\title{
Subleading soft graviton theorem for loop amplitudes
}

\author{
Ashoke Sen \\ Harish-Chandra Research Institute, HBNI, \\ Chhatnag Road, Jhusi, Allahabad 211019, India \\ E-mail: sen@mri.ernet.in
}

ABSTRACT: Superstring field theory gives expressions for heterotic and type II string loop amplitudes that are free from ultraviolet and infrared divergences when the number of non-compact space-time dimensions is five or more. We prove the subleading soft graviton theorem in these theories to all orders in perturbation theory for S-matrix elements of arbitrary number of finite energy external states but only one external soft graviton. We also prove the leading soft graviton theorem for arbitrary number of finite energy external states and arbitrary number of soft gravitons. Since our analysis is based on general properties of one particle irreducible effective action, the results are valid in any theory of quantum gravity that gives finite result for the S-matrix order by order in perturbation theory without violating general coordinate invariance.

KEYwords: Space-Time Symmetries, Superstrings and Heterotic Strings

ARXiV EPRINT: 1703.00024 


\section{Contents}

1 Introduction 1

2 Subleading soft theorem for one external soft graviton 2

$\begin{array}{lll}3 & \text { Leading soft theorem for multiple soft gravitons } & 7\end{array}$

A Justification for dropping Christoffel symbols from covariant derivatives 9

B Derivation of (2.18) 11

\section{Introduction}

In recent years, soft graviton theorem has been studied from various perspectives - perturbative quantum field theory [1-31], perturbative string theory [32-43] and BMS symmetry [44-53]. Our goal in this paper will be to give a general proof of the subleading soft graviton theorem in any perturbative quantum field theory that includes gravity and gives S-matrix elements free from infrared and ultraviolet divergences. At present the only known candidates for such theories are heterotic and type II string field theories [54] in backgrounds with five or more non-compact flat space-time dimensions. ${ }^{1}$

Our strategy will be the same one followed in [55], with the difference that instead of the classical action we work with the one particle irreducible (1PI) effective action. We begin with the gauge invariant 1PI effective action and expand it in powers of all fields including the graviton. We then gauge fix it using a Lorentz covariant gauge fixing condition. The resulting action has manifest Lorentz invariance but not manifest general coordinate invariance. We now introduce the soft graviton field $S_{\mu \nu}$ by covariantizing this action with respect to the soft graviton field. ${ }^{2}$ This requires replacing the background metric by $\eta_{\mu \nu}+2 S_{\mu \nu}$ and the ordinary derivatives by covariant derivatives computed with this background metric. To first subleading order in soft momentum, there are no additional terms coupling the soft graviton to the rest of the fields. Once this replacement is made, we can compute the amplitude involving the soft graviton from the Feynman diagrams of

\footnotetext{
${ }^{1}$ When the number of non-compact space-time dimensions is 4 , the soft graviton theorem is expected to get corrected due to infrared divergences [8,52] unless one uses an unusual definition of the soft limit [11]. Our analysis does not include these theories.

${ }^{2}$ As mentioned in [55], for superstring field theory this procedure would follow from background independence of string field theory that ensures that switching on a soft graviton mode of the string field is equivalent to deforming the background target space metric used for constructing the world-sheet conformal field theory by a soft graviton mode. This is known to be true for bosonic string field theory [56, 57] but has not yet been proven for superstring field theory [58].
} 


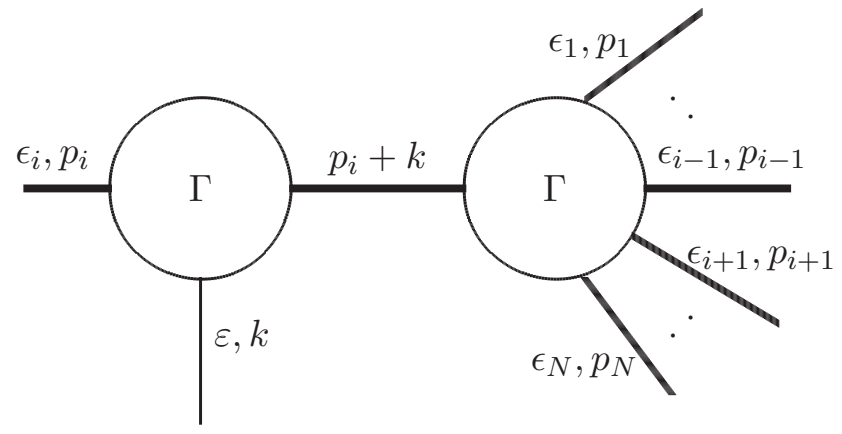

Figure 1. Source of the leading contribution to the string loop amplitude with one external soft graviton. $\epsilon_{i}$ and $p_{i}$ denote the polarization and momentum of the $i$-th external finite energy particles, while $\varepsilon_{\mu \nu}$ and $k$ denote the polarization and momentum of the external soft graviton.

the resulting quantum field theory. Our use of 1PI effective action entails that we need to compute only the tree amplitudes.

The other technical difference from the analysis of [55] is that while covariantizing the action we take all the fields to carry flat tangent space indices instead of curved space indices. This allows us to deal with fermions in the same way as the bosons. We now have to use the vielbein $e_{\mu}^{a}$ instead of the metric to describe the soft graviton field, but to first order in $S_{\mu \nu}$ — which is all we shall need for our analysis - this is done simply by taking $e_{\mu}^{a}$ to be $\delta_{\mu}^{a}+S_{\mu}^{a}$ where the indices are raised and lowered by the flat background metric $\eta$. Since $S_{\mu \nu}=S_{\nu \mu}$, this choice of $e_{\mu}^{a}$ amounts to gauge fixing the local Lorentz symmetry from the beginning and allows us to include superstring field theory in our framework where local Lorentz symmetry is gauge fixed from the beginning.

The rest of the paper is organized as follows. In section 2 we prove the subleading soft graviton theorem for one external soft graviton but arbitrary number of finite energy external states. Some of the technical details of this analysis are given in appendices A and B. In section 3 we prove the leading soft graviton theorem for arbitrary number of soft gravitons and arbitrary number of finite energy external states. In all cases our results are valid to all orders in the perturbation theory.

\section{Subleading soft theorem for one external soft graviton}

In this section we shall prove the subleading soft graviton theorem for amplitudes with one external soft graviton, but arbitrary number of finite energy external states. We begin by describing our notations.

In a Feynman diagram we shall call a line soft if all components of its momentum are small, nearly on-shell if it carries finite energy but satisfies the on-shell condition approximately and hard it is neither soft nor nearly on-shell. We shall work in backgrounds where the number of non-compact space-times dimensions is five or more, and expand the 1PI action in powers of fields around the extremum describing the vacuum solution so that 


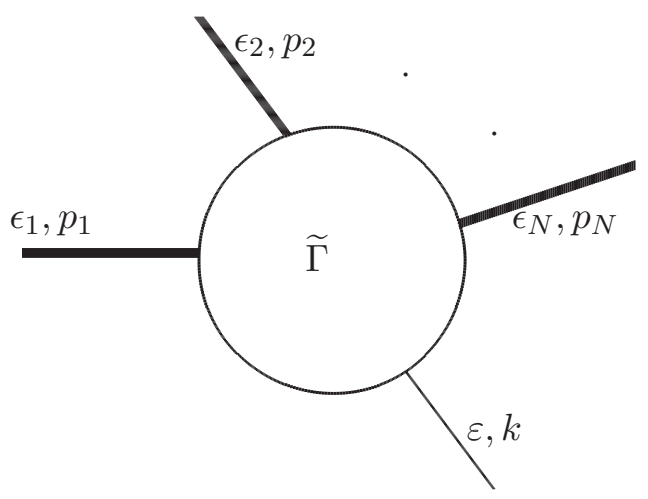

Figure 2. Source of the subleading contribution to the string loop amplitude with one external soft graviton.

there are no tadpoles in the resulting Feynman diagrams. In that case by standard power counting [59] one can show that there are no hidden inverse powers of soft momentum coming from the 1PI vertices with at most one soft external state, even in the presence of massless fields, as long as there are no cubic coupling without derivatives among the massless bosonic fields. Since we shall use the vertices computed from the 1PI effective action, we need to draw only tree graphs, and for this reason there is a clear labelling of each line as soft, nearly on-shell or hard. We use a thin line to denote external soft particle, and a thick line to denote external or internal particles carrying finite momentum and / or energy. All internal lines will denote the full renormalized propagator. We also denote by $\Gamma$ the amputated connected Green's function from which propagators associated with external legs have been removed - for three external legs this coincides with the 1PI vertex.

The leading contribution to the amplitude, carrying one power of soft momentum $k$ in the denominator, comes from the diagrams of the type shown in figure 1 . We shall use the sign convention that all external momenta enter the diagram so that incoming (outgoing) particles carry momentum labels with positive (negative) energy component. If $M_{i}$ denotes the mass of the $i$-th external particle then on-shell condition gives

$$
p_{i}^{2}+M_{i}^{2}=0, \quad k^{2}=0,
$$

together with conditions on polarizations that will be discussed later. Now if we take the internal particle carrying momentum $p_{i}+k$ to have the same mass $M_{i}$, then the propagator gives a terms proportional to $\left\{\left(p_{i}+k\right)^{2}+M_{i}^{2}\right\}^{-1}=\left(2 p_{i} \cdot k\right)^{-1}$. This is responsible for producing the inverse power of soft momentum in the amplitude.

The first subleading contribution in powers of soft momentum comes from the subleading contribution from figure 1 as well as the leading contribution from figure $2 . \widetilde{\Gamma}$ in figure 2 denotes amputated Green's function from which the contributions of the type shown in figure 1 have been subtracted. As a result $\widetilde{\Gamma}$ has no contribution containing inverse powers of momentum.

For computing the contributions from these diagrams we need to determine the coupling of the soft graviton to the rest of the fields. This is done by following the procedure 
outlined in the introduction. We introduce vielbein $e_{\mu}^{a}$ and the inverse vielbein $E_{a}{ }^{\mu}$ in terms of the soft graviton field $S_{\mu \nu}$ to first order in $S_{\mu \nu}$ as

$$
e_{\mu}^{a}=\delta_{\mu}^{a}+S_{\mu}^{a}, \quad E_{a}^{\mu}=\delta_{a}^{\mu}-S_{a}^{\mu},
$$

where all indices are raised and lowered by the flat metric $\eta$. Let $\left\{\Phi_{\alpha}\right\}$ denote the collection of all the fields in the theory, transforming in some large reducible representation of the Lorentz group. ${ }^{3}$ Now in the Lorentz invariant gauge fixed 1PI effective action we replace derivatives of the fields $\Phi_{\alpha}$ as follows:

$$
\partial_{a_{1}} \ldots \partial_{a_{n}} \Phi_{\alpha} \quad \Rightarrow \quad E_{a_{1}}^{\mu_{1}} \ldots E_{a_{n}}^{\mu_{n}} D_{\mu_{1}} \ldots D_{\mu_{n}} \Phi_{\alpha}
$$

where

$$
\mathcal{O}_{1} D_{\mu} \mathcal{O}_{2} \Phi_{\alpha} \equiv \mathcal{O}_{1} \partial_{\mu} \mathcal{O}_{2} \Phi_{\alpha}+\mathcal{O}_{1} \frac{1}{2} \omega_{\mu}^{a b}\left(J_{a b}\right)_{\alpha}^{\gamma} \mathcal{O}_{2} \Phi_{\gamma}, \quad \omega_{\mu}^{a b} \equiv\left(\partial^{b} S_{\mu}{ }^{a}-\partial^{a} S_{\mu}^{b}\right)
$$

Here the $\mathcal{O}_{i}$ 's denote any collection of covariant derivative operators, and $J^{a b}$ are the angular momentum generators, normalized such that if $\Phi$ carries covariant vector indices, then

$$
\left(J^{a b}\right)_{c}{ }^{d}=\delta^{a}{ }_{c} \eta^{b d}-\delta^{b}{ }_{c} \eta^{a d}
$$

Note that in the expression for $D_{\mu}$ in (2.4) we have not included the terms involving the Christoffel symbol $\Gamma_{\mu \nu}^{\rho}$, needed for defining $D_{\mu}$ acting on another covariant derivative $D_{\nu}$ hidden inside $\mathcal{O}_{2}$. We have provided the justification of this in appendix A.

First let us evaluate the contribution from figure 2. This analysis will be more or less identical to the one given in [55]; so we shall be brief. Since we are interested in computing the leading contribution from this graph, we can ignore terms involving $\omega_{\mu}^{a b}$ since they involve derivatives of $S_{\mu \nu}$ and therefore have one or more powers of soft momentum. Therefore for this amplitude the effect of coupling the soft graviton can be obtained by replacing the vielbeins as in (2.2) with $S_{\mu \nu}$ given by the constant polarization tensor $\varepsilon_{\mu \nu}$. This is equivalent to replacing, in the amplitude without the soft graviton, the vielbeins as

$$
e_{\mu}^{a}=\delta_{\mu}^{a}+\varepsilon_{\mu}^{a}, \quad E_{a}^{\mu}=\delta_{a}^{\mu}-\varepsilon_{a}^{\mu} .
$$

Instead of making this replacement inside each vertex and propagator of $\widetilde{\Gamma}$, we can also make this replacement in the final amplitude written in the constant vielbein background. Now since the fields and hence the polarization tensors carry flat tangent space indices, the only place where a vielbein enters in the final expression for the amplitude is in converting the indices of the external momenta $p_{i}$ from space-time indices to flat indices. This can be achieved by using the combination $E_{a}{ }^{\mu} p_{i \mu}=p_{i a}-\varepsilon_{a}^{b} p_{i b}$. Once this is done the indices can be contracted with each other by the metric $\eta$ without any reference to the vielbeins. Therefore the effect of coupling soft graviton in the amplitude in figure 2 is to shift $p_{i a}$ by $-\varepsilon_{a}^{b} p_{i b}$.

\footnotetext{
${ }^{3}$ Even though superstring field theory has infinite number of fields, for any given scattering process we can work with an effective field theory of a finite number of fields by integrating out fields that are sufficiently heavy so that they are not produced in the scattering [54]. Therefore we can assume that the number of fields is finite.
} 
In order to express the result in a convenient form, let us introduce the symbol $\Gamma_{(i)}^{\alpha}\left(p_{i}\right)$ to be the quantity such that

$$
\epsilon_{i, \alpha} \Gamma_{(i)}^{\alpha}\left(p_{i}\right)=\Gamma\left(\epsilon_{1}, p_{1} ; \ldots ; \epsilon_{N}, p_{N}\right),
$$

where the right hand side denotes the amputated N-point Green's function with general off-shell momenta $p_{1}, \ldots, p_{N}$ and polarization tensors $\epsilon_{1}, \ldots, \epsilon_{N}$ without the external soft photon. Therefore the arguments $\epsilon_{1}, p_{1} ; \ldots ; \epsilon_{N}, p_{N}$ other than $\epsilon_{i}, p_{i}$ are hidden in $\Gamma_{(i)}^{\alpha}\left(p_{i}\right)$. With this notation, the result of the previous paragraph can be used to express the amplitude shown in figure 2 as

$$
-\sum_{i=1}^{N} \varepsilon_{a}^{b} p_{i b} \epsilon_{i, \alpha} \frac{\partial}{\partial p_{i a}} \Gamma_{(i)}^{\alpha}\left(p_{i}\right) .
$$

We now turn to the contribution from figure 1. For this we need to study the three point coupling between a single soft graviton and two finite energy particles to the first subleading order in the soft momenta. By our previous argument this may be obtained by covariantizing the quadratic term in the manifestly Lorentz invariant, gauge fixed 1PI effective action without the soft graviton. We begin by writing the general form of the quadratic part of the 1PI effective action in momentum space:

$$
S^{(2)}=\frac{1}{2} \int \frac{d^{D} q_{1}}{(2 \pi)^{D}} \frac{d^{D} q_{2}}{(2 \pi)^{D}} \Phi_{\alpha}\left(q_{1}\right) \mathcal{K}^{\alpha \beta}\left(q_{2}\right) \Phi_{\beta}\left(q_{2}\right)(2 \pi)^{D} \delta^{(D)}\left(q_{1}+q_{2}\right),
$$

where $\Phi_{\alpha}(q)$ now denotes the Fourier transform of the field $\Phi_{\alpha}$ introduced earlier and $D$ is the number of non-compact space-time dimensions. We shall take $\mathcal{K}^{\alpha \beta}(q)$ to be symmetric: ${ }^{4}$

$$
\mathcal{K}^{\alpha \beta}(q)=\mathcal{K}^{\beta \alpha}(-q)
$$

In this case the propagator is given by

$$
D_{F}(q)_{\alpha \beta}=i\left(\mathcal{K}(q)^{-1}\right)_{\alpha \beta},
$$

where $q$ is the momentum flowing from the end carrying the label $\beta$ to the end carrying the label $\alpha$. Noting that the derivative operator $\partial_{\mu}$ in position space becomes a multiplicative operation by $i q_{\mu}$ in the momentum space, and using (2.3), (2.4), we see that effect of coupling a soft graviton field $S_{\mu \nu}=\varepsilon_{\mu \nu} e^{i k . x}$ with

$$
\varepsilon_{\mu \nu}=\varepsilon_{\nu \mu}, \quad k^{\mu} \varepsilon_{\mu \nu}=0=k^{\nu} \varepsilon_{\mu \nu}, \quad \eta^{\mu \nu} \varepsilon_{\mu \nu}=0,
$$

can be obtained by making the following replacement in (2.9):

$$
\begin{aligned}
& \delta^{(D)}\left(q_{1}+q_{2}\right) \mathcal{K}^{\alpha \beta}\left(q_{2}\right) \\
\rightarrow & \delta^{(D)}\left(q_{1}+q_{2}\right) \mathcal{K}^{\alpha \beta}\left(q_{2}\right) \\
- & \delta^{(D)}\left(q_{1}+q_{2}+k\right)\left[\varepsilon_{\mu \nu} q_{2}^{\nu} \frac{\partial}{\partial q_{2 \mu}} \mathcal{K}^{\alpha \beta}\left(q_{2}\right)+\frac{1}{2}\left(k_{a} \varepsilon_{b \mu}-k_{b} \varepsilon_{a \mu}\right) \frac{\partial}{\partial q_{2 \mu}} \mathcal{K}^{\alpha \gamma}\left(q_{2}\right)\left(J^{a b}\right)_{\gamma}^{\beta}\right] .
\end{aligned}
$$

\footnotetext{
${ }^{4}$ For grassmann odd fields there will be an extra minus sign on the right hand side of (2.10), but this does not affect the rest of the analysis.
} 
This gives the part of the action describing the coupling of a soft graviton field $S_{\mu \nu}=$ $\varepsilon_{\mu \nu} e^{i k . x}$ to a pair of other fields to be

$$
\begin{aligned}
S^{(3)}= & \frac{1}{2} \int \frac{d^{D} q_{1}}{(2 \pi)^{D}} \frac{d^{D} q_{2}}{(2 \pi)^{D}}(2 \pi)^{D} \delta^{(D)}\left(q_{1}+q_{2}+k\right) \\
& \times \Phi_{\alpha}\left(q_{1}\right)\left[-\varepsilon_{\mu \nu} q_{2}^{\nu} \frac{\partial}{\partial q_{2 \mu}} \mathcal{K}^{\alpha \beta}\left(q_{2}\right)-\frac{1}{2}\left(k_{a} \varepsilon_{b \mu}-k_{b} \varepsilon_{a \mu}\right) \frac{\partial}{\partial q_{2 \mu}} \mathcal{K}^{\alpha \gamma}\left(q_{2}\right)\left(J^{a b}\right)_{\gamma}^{\beta}\right] \Phi_{\beta}\left(q_{2}\right) .
\end{aligned}
$$

Therefore the three point vertex of a soft graviton of momentum $k$, a $\Phi_{\alpha}$ particle of momentum $p$ and a $\Phi_{\beta}$ particle of momentum $-p-k$ is given by

$$
\begin{aligned}
\Gamma^{(3) \alpha \beta}(\varepsilon, k ; p,-p-k)=\frac{i}{2}[ & -\varepsilon_{\mu \nu}(p+k)^{\nu} \frac{\partial}{\partial p_{\mu}} \mathcal{K}^{\alpha \beta}(-p-k)-\varepsilon_{\mu \nu} p^{\nu} \frac{\partial}{\partial p_{\mu}} \mathcal{K}^{\beta \alpha}(p) \\
& +\frac{1}{2}\left(k_{a} \varepsilon_{b \mu}-k_{b} \varepsilon_{a \mu}\right) \frac{\partial}{\partial p_{\mu}} \mathcal{K}^{\alpha \gamma}(-p-k)\left(J^{a b}\right)_{\gamma}^{\beta} \\
& \left.-\frac{1}{2}\left(k_{a} \varepsilon_{b \mu}-k_{b} \varepsilon_{a \mu}\right) \frac{\partial}{\partial p_{\mu}} \mathcal{K}^{\beta \gamma}(p)\left(J^{a b}\right)_{\gamma}^{\alpha}\right]
\end{aligned}
$$

The contribution from the amplitude shown in figure 1 may now be expressed as

$$
\epsilon_{i, \alpha} \Gamma^{(3) \alpha \beta}\left(\varepsilon, k ; p_{i},-p_{i}-k\right) i\left\{\mathcal{K}^{-1}\left(-p_{i}-k\right)\right\}_{\beta \delta} \Gamma_{(i)}^{\delta}\left(p_{i}+k\right),
$$

where $\Gamma_{(i)}$ has been defined in (2.7). It has been shown in appendix B that as long as $\epsilon_{i, \alpha}$ and $p_{i}$ satisfy the on-shell condition

$$
\epsilon_{i, \alpha} \mathcal{K}^{\alpha \beta}\left(-p_{i}\right)=0
$$

(2.16) can be reduced to

$$
\begin{aligned}
& \left(p_{i} \cdot k\right)^{-1} \varepsilon_{\mu \nu} p_{i}^{\mu} p_{i}^{\nu} \epsilon_{i, \alpha} \Gamma_{(i)}^{\alpha}\left(p_{i}\right)+\left(p_{i} \cdot k\right)^{-1} \varepsilon_{\mu \nu} p_{i}^{\mu} p_{i}^{\nu} \epsilon_{i, \alpha} k_{\rho} \frac{\partial}{\partial p_{i \rho}} \Gamma_{(i)}^{\alpha}\left(p_{i}\right) \\
& \quad+\left(p_{i} \cdot k\right)^{-1} k_{a} \varepsilon_{b \mu} p_{i}^{\mu} \epsilon_{i, \alpha}\left(J^{a b}\right)_{\gamma}^{\alpha} \Gamma_{(i)}^{\gamma}\left(p_{i}\right) .
\end{aligned}
$$

After summing over $i$ and adding the contribution (2.8) from figure 2 we get the subleading soft graviton theorem for one soft graviton:

$$
\begin{aligned}
\Gamma\left(\varepsilon, k ; \epsilon_{1}, p_{1} ; \ldots ; \epsilon_{N}, p_{N}\right)= & \sum_{i=1}^{N}\left(p_{i} \cdot k\right)^{-1} \varepsilon_{\mu \nu} p_{i}^{\mu} p_{i}^{\nu} \epsilon_{i, \alpha} \Gamma_{(i)}^{\alpha}\left(p_{i}\right) \\
& +\sum_{i=1}^{N}\left\{\left(p_{i} \cdot k\right)^{-1} \varepsilon_{\mu \nu} p_{i}^{\mu} p_{i}^{\nu} k_{\rho}-\varepsilon_{\rho b} p_{i}^{b}\right\} \epsilon_{i, \alpha} \frac{\partial}{\partial p_{i \rho}} \Gamma_{(i)}^{\alpha}\left(p_{i}\right) \\
& +\sum_{i=1}^{N}\left(p_{i} \cdot k\right)^{-1} k_{a} \varepsilon_{b \mu} p_{i}^{\mu} \epsilon_{i, \alpha}\left(J^{a b}\right)_{\gamma}^{\alpha} \Gamma_{(i)}^{\gamma}\left(p_{i}\right)
\end{aligned}
$$

with $\Gamma_{(i)}^{\gamma}\left(p_{i}\right)$ defined through (2.7). In order to compare with the result of [6] we rewrite this as

$$
\begin{aligned}
& \Gamma\left(\varepsilon, k ; \epsilon_{1}, p_{1} ; \ldots ; \epsilon_{N}, p_{N}\right)=\sum_{i=1}^{N}\left(p_{i} \cdot k\right)^{-1} \varepsilon_{\mu \nu} p_{i}^{\mu} p_{i}^{\nu} \epsilon_{i, \alpha} \Gamma_{(i)}^{\alpha}\left(p_{i}\right) \\
& \quad+\sum_{i=1}^{N}\left(p_{i} \cdot k\right)^{-1} \varepsilon_{\mu \nu} p_{i}^{\mu} k_{\rho} \epsilon_{i, \alpha}\left[\delta_{\gamma}^{\alpha}\left\{p_{i}^{\nu} \frac{\partial}{\partial p_{i \rho}}-p_{i}^{\rho} \frac{\partial}{\partial p_{i \nu}}\right\}+\left(J^{\rho \nu}\right)_{\gamma}^{\alpha}\right] \Gamma_{(i)}^{\gamma}\left(p_{i}\right)
\end{aligned}
$$




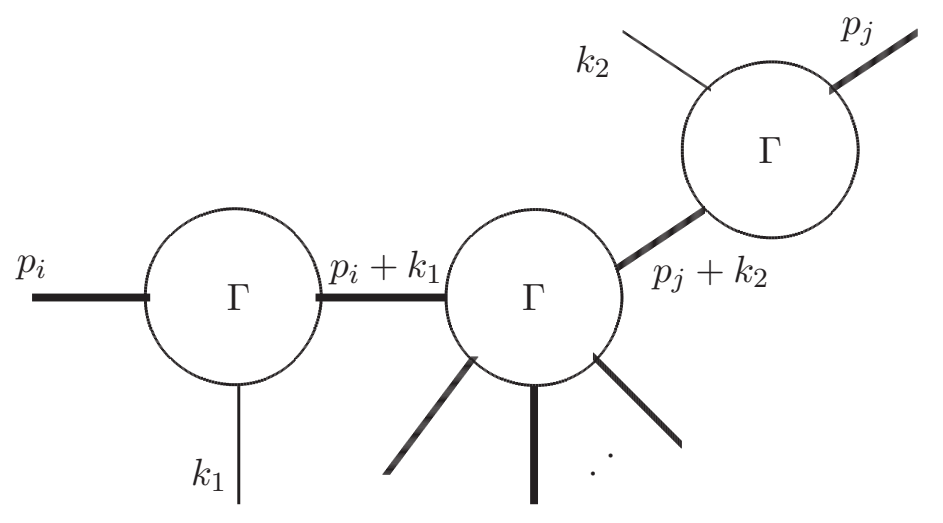

Figure 3. Two external soft gravitons attached to different external lines carrying finite momenta.

This is the standard form of the subleading soft graviton theorem given in [6] with the term inside the curly bracket representing orbital angular momentum operator and $J^{\rho \nu}$ representing the spin angular momentum operator.

It was shown in [14] that in certain theories the soft graviton amplitude is determined by the form of the on-shell three point vertex of one soft graviton and a pair of finite energy particles. Our analysis shows that the subleading soft graviton amplitude is in fact completely determined in terms of momentum and spin of the external finite energy particles, and no further information on the three point function is required.

\section{Leading soft theorem for multiple soft gravitons}

We shall now consider amplitudes with multiple soft gravitons. A general form of the leading order amplitude with multiple soft gravitons was given in [2]. This analysis was based on the structure of the on-shell three point function of a soft graviton and a pair of finite energy particles of arbitrary mass and spin, which in turn was derived in [1]. However the analysis of [1] was done in four space-time dimensions in which the graviton polarization tensor of a given helicity can be represented as the square of the photon polarization vector of the same helicity. We believe that with a little effort it may be possible to generalize this proof to arbitrary dimensions, but we shall follow a different approach based on the general relation between the off-shell three point functions and off-shell two point functions given in (2.15).

We shall first analyze the case where we have two soft external gravitons carrying momenta $k_{1}$ and $k_{2}$. In this case the leading contribution has two powers of soft momenta in the denominator, arising from diagrams where the two soft gravitons attach to different external legs as in figure 3 or both soft gravitons attach to the same external leg as in figure 4 . In either of the diagrams, the product of the leading contributions from the three point vertex and the internal propagator that follows it is given by

$$
\varepsilon_{\mu \nu} p_{i}^{\mu} p_{i}^{\nu}\left(p_{i} \cdot \ell\right)^{-1}
$$




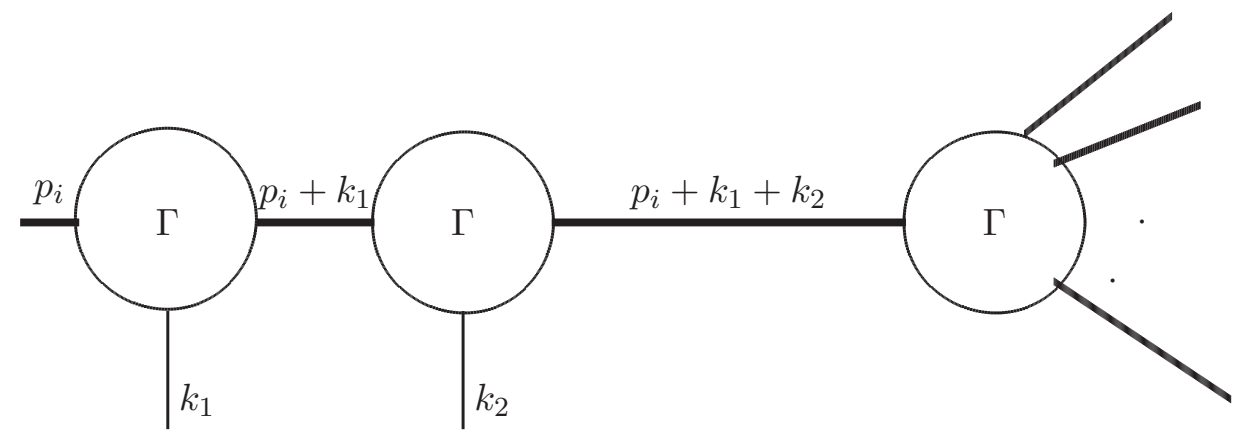

Figure 4. Two external soft gravitons attached to the same external line carrying finite momenta.

where $p_{i}$ is the (nearly) on-shell momentum entering the vertex, $\varepsilon$ is the polarization of the soft graviton and $p_{i}+\ell$ is the momentum carried by the internal propagator that follows the vertex. The derivation of this is identical to the derivation of the first term on the right hand side of (2.18) and follows easily from the analysis given in appendix B.

From this point onwards the analysis proceeds as in [55]. The contribution from figure 3 takes the form

$$
\frac{1}{p_{i} \cdot k_{1}} \varepsilon_{\mu \nu}^{(1)} p_{i}^{\mu} p_{i}^{\nu} \times \frac{1}{p_{j} \cdot k_{2}} \varepsilon_{\rho \sigma}^{(2)} p_{j}^{\rho} p_{j}^{\sigma} \times \Gamma\left(\epsilon_{1}, p_{1} ; \ldots ; \epsilon_{N}, p_{N}\right)+\text { less singular terms } .
$$

On the other hand the contribution from figure 4 takes the form

$$
\frac{1}{p_{i} \cdot k_{1}} \varepsilon_{\mu \nu}^{(1)} p_{i}^{\mu} p_{i}^{\nu} \times \frac{1}{p_{i} \cdot\left(k_{1}+k_{2}\right)} \varepsilon_{\rho \sigma}^{(2)} p_{j}^{\rho} p_{j}^{\sigma} \times \Gamma\left(\epsilon_{1}, p_{1} ; \ldots ; \epsilon_{N}, p_{N}\right)+\text { less singular terms } .
$$

There is another contribution where the external soft lines carrying momenta $k_{1}$ and $k_{2}$ are exchanged in (3.3). Adding this to (3.3) we get

$$
\frac{1}{p_{i} \cdot k_{1}} \varepsilon_{\mu \nu}^{(1)} p_{i}^{\mu} p_{i}^{\nu} \times \frac{1}{p_{i} \cdot k_{2}} \varepsilon_{\rho \sigma}^{(2)} p_{i}^{\rho} p_{i}^{\sigma} \times \Gamma\left(\epsilon_{1}, p_{1} ; \ldots ; \epsilon_{N}, p_{N}\right)+\text { less singular terms } .
$$

After summing over all possible insertions of the two soft gravitons on $N$ external lines carrying finite momentum, we get [2]

$$
\sum_{i=1}^{N} \frac{1}{p_{i} \cdot k_{1}} \varepsilon_{\mu \nu}^{(1)} p_{i}^{\mu} p_{i}^{\nu} \times \sum_{j=1}^{N} \frac{1}{p_{j} \cdot k_{2}} \varepsilon_{\rho \sigma}^{(2)} p_{j}^{\rho} p_{j}^{\sigma} \times \Gamma\left(\epsilon_{1}, p_{1} ; \ldots ; \epsilon_{N}, p_{N}\right)+\text { less singular terms } .
$$

For $m$ external soft gravitons the leading term will have $m$ powers of soft momentum in the denominator, coming from diagrams where each external soft graviton gets attached to a nearly on-shell line. After summing over all possible insertions we arrive at the generalization of (3.5):

$\begin{aligned} \Gamma\left(\varepsilon^{(1)}, k_{1} ; \ldots ; \varepsilon^{(m)}, k_{m} ; \epsilon_{1}, p_{1} ; \ldots ; \epsilon_{N}, p_{N}\right)= & \prod_{s=1}^{m}\left[\sum_{i=1}^{N} \frac{1}{p_{i} \cdot k_{s}} \varepsilon_{\mu \nu}^{(s)} p_{i}^{\mu} p_{i}^{\nu}\right] \Gamma\left(\epsilon_{1}, p_{1} ; \ldots ; \epsilon_{N}, p_{N}\right) \\ & + \text { less singular terms . }\end{aligned}$ 


\section{Acknowledgments}

This work was supported in part by the DAE project 12-R\&D-HRI-5.02-0303 and J. C. Bose fellowship of the Department of Science and Technology, India.

\section{A Justification for dropping Christoffel symbols from covariant deriva- tives}

In defining the covariant derivative operator $D_{\mu}$ appearing in (2.3) using (2.4), we dropped possible terms involving the Christoffel symbol $\Gamma_{\mu \nu}^{\rho}$. These Christoffel symbols arise when $D_{\mu}$ acts on another covariant derivative $D_{\nu}$. In this appendix we shall justify this.

The relevant place where the appearance of these Christoffel symbol terms could affect our analysis is in (2.14). Let us suppose that we have a term in the action of the form

$$
\int d^{D} x E_{c_{1}}^{\mu_{1}} \cdots E_{c_{r}}^{\mu_{r}} \Phi_{a_{1} \cdots a_{m}} D_{\mu_{1}} \cdots D_{\mu_{r}} \Psi_{b_{1} \cdots b_{n}}
$$

with the free indices $a_{i}, b_{i}, c_{i}$ contracted with the flat metric $\eta$. Here $E_{c}{ }^{\mu}$ are the inverse vielbeins and $\Phi$ and $\Psi$ are appropriate tensor fields carrying tangent space indices. Upon expressing $D_{\mu_{i}}$ as $\partial_{\mu_{i}}+\cdots$, a typical term in $\cdots$ that was ignored in (2.4) is of the form

$$
-\int d^{D} x E_{c_{1}}^{\mu_{1}} \cdots E_{c_{r}}^{\mu_{r}} \Phi_{a_{1} \cdots a_{m}} D_{\mu_{1}} \cdots D_{\mu_{i-1}} \Gamma_{\mu_{i} \mu_{j}}^{\rho} D_{\mu_{i+1}} \cdots D_{\mu_{j-1}} D_{\rho} D_{\mu_{j+1}} \cdots D_{\mu_{r}} \Psi_{b_{1} \cdots b_{n}} .
$$

In the soft limit $\Gamma_{\mu_{i} \mu_{j}}^{\rho}$ carries a factor of the soft momentum and a factor of soft graviton polarization. Therefore we can replace the rest of the covariant derivatives by ordinary derivatives, ignore terms involving derivatives of $\Gamma$ and replace $E_{c}{ }^{\mu}$ by $\delta_{c}{ }^{\mu}$. With this (A.2) reduces to

$$
-\int d^{D} x \delta_{c_{1}}^{\mu_{1}} \cdots \delta_{c_{r}}^{\mu_{r}} \Gamma_{\mu_{i} \mu_{j}}^{\rho} \Phi_{a_{1} \cdots a_{m}} \partial_{\rho} \partial_{\mu_{1}} \cdots \partial_{\mu_{i-1}} \partial_{\mu_{i+1}} \cdots \partial_{\mu_{j-1}} \partial_{\mu_{j+1}} \cdots \partial_{\mu_{r}} \Psi_{b_{1} \cdots b_{n}} .
$$

Let us now return to (A.1) and, using integration by parts, express this as

$$
(-1)^{r} \int d^{D} x E_{c_{1}}^{\mu_{1}} \cdots E_{c_{r}}^{\mu_{r}} \Psi_{b_{1} \cdots b_{n}} D_{\mu_{1}} \cdots D_{\mu_{r}} \Phi_{a_{1} \cdots a_{m}} .
$$

Note that integration by parts will reverse the order in which the covariant derivatives act, but since the commutator of two covariant derivatives is proportional to the Riemann tensor and carries two powers of soft momentum, we can ignore the reversal of order. For grassmann odd fields there will be an additional minus sign in (A.4), but this will cancel with an additional minus sign that will appear in going from (A.5) to (A.6). The fields will also carry spinor indices contracted with appropriate Lorentz covariant tensors, but this does not affect the analysis. By expanding the expression for $D_{\mu_{i}}$ in (A.4) we shall get the analog of (A.2), and from this the analog of (A.3):

$$
-(-1)^{r} \int d^{D} x \delta_{c_{1}}^{\mu_{1}} \cdots \delta_{c_{r}}^{\mu_{r}} \Gamma_{\mu_{i} \mu_{j}}^{\rho} \Psi_{b_{1} \cdots b_{n}} \partial_{\rho} \partial_{\mu_{1}} \cdots \partial_{\mu_{i-1}} \partial_{\mu_{i+1}} \cdots \partial_{\mu_{j-1}} \partial_{\mu_{j+1}} \cdots \partial_{\mu_{r}} \Phi_{a_{1} \cdots a_{m}} .
$$


We shall now again integrate by parts and ignore derivatives of $\Gamma$ since that will generate two powers of soft momentum. This takes (A.5) to

$$
-(-1)^{r}(-1)^{r-1} \int d^{D} x \delta_{c_{1}}^{\mu_{1}} \cdots \delta_{c_{r}}^{\mu_{r}} \Gamma_{\mu_{i} \mu_{j}}^{\rho} \Phi_{a_{1} \cdots a_{m}} \partial_{\rho} \partial_{\mu_{1}} \cdots \partial_{\mu_{i-1}} \partial_{\mu_{i+1}} \cdots \partial_{\mu_{j-1}} \partial_{\mu_{j+1}} \cdots \partial_{\mu_{r}} \Psi_{b_{1} \cdots b_{n}} .
$$

We now see that (A.3) and (A.6) cancel each other. This shows that once we express (A.1) as

$$
\frac{1}{2} \int d^{D} x E_{c_{1}}^{\mu_{1}} \cdots E_{c_{r}}^{\mu_{r}}\left[\Phi_{a_{1} \cdots a_{m}} D_{\mu_{1}} \cdots D_{\mu_{r}} \Psi_{b_{1} \cdots b_{n}}+(-1)^{r} \Psi_{b_{1} \cdots b_{n}} D_{\mu_{r}} \cdots D_{\mu_{1}} \Phi_{a_{1} \cdots a_{m}}\right],
$$

the terms involving Christoffel symbols drop out.

The alert reader may worry that the above derivation assumes that the two point function computed from the 1PI action has the form of a polynomial in derivatives while in practice this is not so. We can allay this fear by working in momentum space. Suppose that in the absence of the soft graviton, the quadratic term of the 1PI effective action involving single powers of $\Phi$ and $\Psi$ takes the form

$$
\int \frac{d^{D} p}{(2 \pi)^{D}} \Phi_{a_{1} \cdots a_{m}}(-p) f_{c_{1} \cdots c_{r}}(p) \Psi_{b_{1} \cdots b_{n}}(p),
$$

contracted with $\eta$ 's. Here $\Phi$ and $\Psi$ are Fourier transforms of the fields that appear in (A.1) and $f$ is some function of the momentum $p$. Then after coupling to the soft graviton, the unwanted terms given in (A.3) have the form

$$
-\frac{1}{2} \int \frac{d^{D} p}{(2 \pi)^{D}} \Phi_{a_{1} \cdots a_{m}}(-p-k) \frac{\partial^{2} f_{c_{1} \cdots c_{r}}(p)}{\partial p_{\mu} \partial p_{\nu}} \Psi_{b_{1} \cdots b_{n}}(p)\left(-i p_{\rho}\right) \Gamma_{\mu \nu}^{\rho}(k),
$$

where $\Gamma$ now denotes the Christoffel symbol computed using soft graviton in the momentum space. In arriving at (A.9) we have used the fact that in momentum space $\partial_{\mu}$ is replaced by $i p_{\mu}$.

Now by making a $p \rightarrow-p$ change of variables in (A.8) we arrive at a similar formula with the $\Psi$ and $\Phi$ exchanged

$$
\int \frac{d^{D} p}{(2 \pi)^{D}} \Psi_{b_{1} \cdots b_{n}}(-p) f_{c_{1} \cdots c_{r}}(-p) \Phi_{a_{1} \cdots a_{m}}(p),
$$

Its covariantization will generate the analog of (A.9)

$$
-\frac{1}{2} \int \frac{d^{D} p}{(2 \pi)^{D}} \Psi_{b_{1} \cdots b_{m}}(-p-k) \frac{\partial^{2} f_{c_{1} \cdots c_{r}}(-p)}{\partial p_{\mu} \partial p_{\nu}} \Phi_{a_{1} \cdots n_{n}}(p)\left(-i p_{\rho}\right) \Gamma_{\mu \nu}^{\rho}(k) .
$$

Now making a change of variables $p \rightarrow-p-k$ we get

$$
-\frac{1}{2} \int \frac{d^{D} p}{(2 \pi)^{D}} \Phi_{a_{1} \cdots n_{n}}(-p-k) \frac{\partial^{2} f_{c_{1} \cdots c_{r}}(p+k)}{\partial p_{\mu} \partial p_{\nu}} \Psi_{b_{1} \cdots b_{m}}(p)\left(i\left(p_{\rho}+k_{\rho}\right)\right) \Gamma_{\mu \nu}^{\rho}(k) .
$$

Averaging over (A.9) and (A.12), and using the fact that $\Gamma$ already contains one power of soft momentum, we now easily see that the integrand has two powers of $k$. Therefore it vanishes to the first subleading order in the soft momentum $k$. 


\section{B Derivation of (2.18)}

Our goal in this appendix will be to prove the equality of (2.16) and (2.18). We begin by studying some properties of the matrix $\mathcal{K}^{\alpha \beta}(q)$ appearing in the kinetic term (2.9), and the propagator $D_{F}$ defined in (2.11). Let us also define $\Xi(q)$ via

$$
\Xi(q)=\left(q^{2}+M^{2}\right) D_{F}(q)=i\left(q^{2}+M^{2}\right) \mathcal{K}(q)^{-1},
$$

where $M$ is the mass of the external state that we shall be interested in. $\Xi(q)$ obviously depends on $M$, but this dependence is not displayed explicitly. At a generic value of $q$, $\Xi(q)$ has the same rank as that of $\mathcal{K}(q)$ or $D_{F}(q)$, i.e. the total number of fields. But in the limit $q^{2}+M^{2} \rightarrow 0$, we expect $\Xi(q)$ to approach a finite matrix of rank that is typically less than the total number of fields, since only a subset of particles have mass $M$ producing a pole in the propagator $D_{F}$ at $q^{2}+M^{2}=0 .{ }^{5}$

Using (B.1) we get

$$
\mathcal{K}(q) \Xi(q)=i\left(q^{2}+M^{2}\right) .
$$

Differentiation of both sides of (B.2) with respect to $q_{\mu}$ gives

$$
\frac{\partial \mathcal{K}(q)}{\partial q_{\mu}} \Xi(q)+\mathcal{K}(q) \frac{\partial \Xi(q)}{\partial q_{\mu}}=2 i q^{\mu} .
$$

Now suppose $\epsilon_{\alpha}$ denotes the polarization of an on-shell state carrying momentum $q$ and mass $M$. Then we have

$$
\epsilon_{\alpha} \mathcal{K}^{\alpha \beta}(q)=0, \quad \text { at } q^{2}+M^{2}=0 .
$$

Combining this with (B.3) we get

$$
\epsilon_{\alpha}\left[\frac{\partial \mathcal{K}(q)}{\partial q_{\mu}} \Xi(q)\right]_{\gamma}^{\alpha}=2 i \epsilon_{\gamma} q_{\mu} \quad \text { at } q^{2}+M^{2}=0 .
$$

Next we shall study the consequence of Lorentz invariance. First of all, since we use a Lorentz covariant gauge fixing condition, the matrix $\mathcal{K}$ and $\Xi$ must be Lorentz covariant:

$$
\begin{aligned}
\mathcal{K}^{\alpha \gamma}(q)\left(J^{a b}\right)_{\gamma}^{\beta}+\mathcal{K}^{\gamma \beta}(q)\left(J^{a b}\right)_{\gamma}^{\alpha}=q^{a} \frac{\partial \mathcal{K}^{\alpha \beta}(q)}{\partial q_{b}}-q^{b} \frac{\partial \mathcal{K}^{\alpha \beta}(q)}{\partial q_{a}}, \\
-\Xi_{\alpha \gamma}(q)\left(J^{a b}\right)_{\beta}^{\gamma}-\Xi_{\gamma \beta}(q)\left(J^{a b}\right)_{\alpha}^{\gamma}=q^{a} \frac{\partial \Xi_{\alpha \beta}(q)}{\partial q_{b}}-q^{b} \frac{\partial \Xi_{\alpha \beta}(q)}{\partial q_{a}} .
\end{aligned}
$$

It is easy to see, using (B.5), (B.6) and (B.7) that at $q^{2}+M^{2}=0$,

$$
\begin{aligned}
\epsilon_{\alpha}\left(J^{a b}\right)_{\beta}^{\alpha}\left[\frac{\partial \mathcal{K}(q)}{\partial q_{\mu}} \Xi(q)\right]_{\gamma}^{\beta}= & \epsilon_{\beta}\left[\left(q^{a} \frac{\partial^{2} \mathcal{K}(q)}{\partial q_{b} \partial q_{\mu}}-q^{b} \frac{\partial^{2} \mathcal{K}}{\partial q_{a} \partial q_{\mu}}\right) \Xi(q)\right]_{\gamma}^{\beta} \\
& +\epsilon_{\beta}\left[\frac{\partial \mathcal{K}(q)}{\partial q_{\mu}}\left(q^{a} \frac{\partial \Xi(q)}{\partial q_{b}}-q^{b} \frac{\partial \Xi(q)}{\partial q_{a}}\right)\right]_{\gamma}^{\beta} \\
& +2 i q^{\mu} \epsilon_{\alpha}\left(J^{a b}\right)_{\gamma}^{\alpha}-2 i \epsilon_{\gamma}\left(q^{a} \eta^{\mu b}-q^{b} \eta^{\mu a}\right) .
\end{aligned}
$$

\footnotetext{
${ }^{5}$ For massless particles the propagator may have double poles in some gauges, e.g. in a generic covariant gauge the propagator of a massless gauge field is given by $\left(\eta^{\mu \nu}-\beta k^{\mu} k^{\nu} / k^{2}\right) / k^{2}$ for some constant $\beta$. We shall assume that our gauge fixing condition is such that we avoid propagators with double poles.
} 
Next we turn to the analysis of the three point vertex $\Gamma^{(3) \alpha \beta}(\varepsilon, k ; p,-p-k)$ given in (2.15). Using (B.6) we can simplify the second line of (2.15) and get

$$
\begin{aligned}
\Gamma^{(3) \alpha \beta}(\varepsilon, k ; p,-p-k)= & \frac{i}{2}\left[-\varepsilon_{\mu \nu}(p+k)^{\nu} \frac{\partial \mathcal{K}^{\alpha \beta}(-p-k)}{\partial p_{\mu}}-\varepsilon_{\mu \nu} p^{\nu} \frac{\partial \mathcal{K}^{\beta \alpha}(p)}{\partial p_{\mu}}\right. \\
& -\frac{1}{2}\left(k_{a} \varepsilon_{b \mu}-k_{b} \varepsilon_{a \mu}\right) \frac{\partial \mathcal{K}^{\gamma \beta}(-p-k)}{\partial p_{\mu}}\left(J^{a b}\right)_{\gamma}^{\alpha} \\
& +\frac{1}{2}\left(k_{a} \varepsilon_{b \mu}-k_{b} \varepsilon_{a \mu}\right) \frac{\partial}{\partial p_{\mu}}\left\{p^{a} \frac{\partial \mathcal{K}^{\alpha \beta}(-p-k)}{\partial p_{b}}-p^{b} \frac{\partial \mathcal{K}^{\alpha \beta}(-p-k)}{\partial p_{a}}\right\} \\
& \left.-\frac{1}{2}\left(k_{a} \varepsilon_{b \mu}-k_{b} \varepsilon_{a \mu}\right) \frac{\partial \mathcal{K}^{\beta \gamma}(p)}{\partial p_{\mu}}\left(J^{a b}\right)_{\gamma}^{\alpha}\right] .
\end{aligned}
$$

Using (2.12), (2.10), expanding $\mathcal{K}^{\alpha \beta}(-p-k)$ in the first term in a Taylor series expansion in $k_{\rho}$, and keeping terms up to first subleading order in the soft momentum $k$, we can express (B.9) as

$$
\begin{aligned}
\Gamma^{(3) \alpha \beta}(\varepsilon, k ; p,-p-k)= & \frac{i}{2}\left[-2 \varepsilon_{\mu \nu} p^{\nu} \frac{\partial \mathcal{K}^{\alpha \beta}(-p)}{\partial p_{\mu}}-2 \varepsilon_{\mu \nu} p^{\nu} k_{\sigma} \frac{\partial^{2} \mathcal{K}^{\alpha \beta}(-p)}{\partial p_{\sigma} \partial p_{\mu}}\right. \\
& \left.+k \cdot p \varepsilon_{b \mu} \frac{\partial^{2} \mathcal{K}^{\alpha \beta}(-p)}{\partial p_{\mu} \partial p_{b}}-\left(k_{a} \varepsilon_{b \mu}-k_{b} \varepsilon_{a \mu}\right) \frac{\partial \mathcal{K}^{\gamma \beta}(-p)}{\partial p_{\mu}}\left(J^{a b}\right)_{\gamma}^{\alpha}\right] .
\end{aligned}
$$

We now turn to (2.16). Substituting (B.10) into (2.16) we get the net contribution to (2.16) to first subleading order in the soft momentum:

$$
\begin{aligned}
& \epsilon_{i, \alpha} \Gamma^{(3) \alpha \beta}\left(\varepsilon, k ; p_{i},-p_{i}-k\right) i\left\{\mathcal{K}^{-1}\left(-p_{i}-k\right)\right\}_{\beta \delta} \Gamma_{(i)}^{\delta}\left(p_{i}+k\right) \\
&= \frac{1}{2} \epsilon_{i, \alpha}\left[2 \varepsilon_{\mu \nu} p_{i}^{\nu} \frac{\partial \mathcal{K}^{\alpha \beta}\left(-p_{i}\right)}{\partial p_{i \mu}}+2 \varepsilon_{\mu \nu} p_{i}^{\nu} k_{\sigma} \frac{\partial^{2} \mathcal{K}^{\alpha \beta}\left(-p_{i}\right)}{\partial p_{i \sigma} \partial p_{i \mu}}-k \cdot p_{i} \varepsilon_{b \mu} \frac{\partial^{2} \mathcal{K}^{\alpha \beta}\left(-p_{i}\right)}{\partial p_{i \mu} \partial p_{i b}}\right. \\
&\left.\quad+\left(k_{a} \varepsilon_{b \mu}-k_{b} \varepsilon_{a \mu}\right) \frac{\partial \mathcal{K}^{\gamma \beta}\left(-p_{i}\right)}{\partial p_{i \mu}}\left(J^{a b}\right)_{\gamma}^{\alpha}\right]\left\{\mathcal{K}^{-1}\left(-p_{i}-k\right)\right\}_{\beta \delta}\left[1+k_{\rho} \frac{\partial}{\partial p_{i \rho}}\right] \Gamma_{(i)}^{\delta}\left(p_{i}\right) .
\end{aligned}
$$

Replacing the $\mathcal{K}^{-1}\left(-p_{i}-k\right)$ factor using (B.2) after setting $M=M_{i}$, the mass of the $i$-th external state, and using $\left(p_{i}+k\right)^{2}+M_{i}^{2}=2 p_{i} \cdot k$, we may express (B.11) as

$$
\begin{aligned}
& -\frac{i}{2}\left(2 p_{i} \cdot k\right)^{-1} \epsilon_{i, \alpha}\left[2 \varepsilon_{\mu \nu} p_{i}^{\nu} \frac{\partial \mathcal{K}^{\alpha \beta}\left(-p_{i}\right)}{\partial p_{i \mu}}+2 \varepsilon_{\mu \nu} p_{i}^{\nu} k_{\sigma} \frac{\partial^{2} \mathcal{K}^{\alpha \beta}\left(-p_{i}\right)}{\partial p_{i \sigma} \partial p_{i \mu}}-k \cdot p_{i} \varepsilon_{b \mu} \frac{\partial^{2} \mathcal{K}^{\alpha \beta}\left(-p_{i}\right)}{\partial p_{i \mu} \partial p_{i b}}\right. \\
& \left.+\left(k_{a} \varepsilon_{b \mu}-k_{b} \varepsilon_{a \mu}\right) \frac{\partial \mathcal{K}^{\gamma \beta}\left(-p_{i}\right)}{\partial p_{i \mu}}\left(J^{a b}\right)_{\gamma}^{\alpha}\right] \Xi_{\beta \delta}\left(-p_{i}-k\right)\left[1+k_{\rho} \frac{\partial}{\partial p_{i \rho}}\right] \Gamma_{(i)}^{\delta}\left(p_{i}\right) \\
& =-\frac{i}{2}\left(2 p_{i} \cdot k\right)^{-1} \epsilon_{i, \alpha}\left[2 \varepsilon_{\mu \nu} p_{i}^{\nu} \frac{\partial \mathcal{K}^{\alpha \beta}\left(-p_{i}\right)}{\partial p_{i \mu}}+2 \varepsilon_{\mu \nu} p_{i}^{\nu} k_{\sigma} \frac{\partial^{2} \mathcal{K}^{\alpha \beta}\left(-p_{i}\right)}{\partial p_{i \sigma} \partial p_{i \mu}}-k \cdot p_{i} \varepsilon_{b \mu} \frac{\partial^{2} \mathcal{K}^{\alpha \beta}\left(-p_{i}\right)}{\partial p_{i \mu} \partial p_{i b}}\right. \\
& \left.+\left(k_{a} \varepsilon_{b \mu}-k_{b} \varepsilon_{a \mu}\right) \frac{\partial \mathcal{K}^{\gamma \beta}\left(-p_{i}\right)}{\partial p_{i \mu}}\left(J^{a b}\right)_{\gamma}^{\alpha}\right]\left\{\Xi_{\beta \delta}\left(-p_{i}\right)+k_{\sigma} \frac{\partial \Xi_{\beta \delta}\left(-p_{i}\right)}{\partial p_{i \sigma}}\right\}\left[1+k_{\rho} \frac{\partial}{\partial p_{i \rho}}\right] \Gamma_{(i)}^{\delta}\left(p_{i}\right) .
\end{aligned}
$$


If $p_{i}^{2}+M_{i}^{2}=0$, then the $\epsilon_{i, \alpha}$ in (B.12) is a physical state of mass $M_{i}$. Therefore we can now use (B.5) to express (B.12) as

$$
\begin{aligned}
& \left(p_{i} \cdot k\right)^{-1} \varepsilon_{\mu \nu} p_{i}^{\mu} p_{i}^{\nu} \epsilon_{i, \alpha} \Gamma_{(i)}^{\alpha}\left(p_{i}\right)-\frac{i}{2}\left(p_{i} \cdot k\right)^{-1} \epsilon_{i, \alpha} \varepsilon_{\mu \nu} p_{i}^{\nu} k_{\sigma} \frac{\partial^{2} \mathcal{K}^{\alpha \beta}\left(-p_{i}\right)}{\partial p_{i \sigma} \partial p_{i \mu}} \Xi_{\beta \delta}\left(-p_{i}\right) \Gamma_{(i)}^{\delta}\left(p_{i}\right) \\
& +\frac{i}{4} \epsilon_{i, \alpha} \varepsilon_{b \mu} \frac{\partial^{2} \mathcal{K}^{\alpha \beta}\left(-p_{i}\right)}{\partial p_{i \mu} \partial p_{i b}} \Xi_{\beta \delta}\left(-p_{i}\right) \Gamma_{(i)}^{\delta}\left(p_{i}\right) \\
& -i \epsilon_{i, \alpha}\left(2 p_{i} \cdot k\right)^{-1} \varepsilon_{\mu \nu} p_{i}^{\nu} \frac{\partial \mathcal{K}^{\alpha \beta}\left(-p_{i}\right)}{\partial p_{i \mu}} k_{\rho} \frac{\partial \Xi_{\beta \delta}\left(-p_{i}\right)}{\partial p_{i \rho}} \Gamma_{(i)}^{\delta}\left(p_{i}\right) \\
& +\left(p_{i} \cdot k\right)^{-1} \varepsilon_{\mu \nu} p_{i}^{\mu} p_{i}^{\nu} \epsilon_{i, \alpha} k_{\rho} \frac{\partial}{\partial p_{i \rho}} \Gamma_{(i)}^{\alpha}\left(p_{i}\right) \\
& -\frac{i}{4}\left(p_{i} \cdot k\right)^{-1} \epsilon_{i, \alpha}\left(k_{a} \varepsilon_{b \mu}-k_{b} \varepsilon_{a \mu}\right) \frac{\partial \mathcal{K}^{\gamma \beta}\left(-p_{i}\right)}{\partial p_{i \mu}}\left(J^{a b}\right)_{\gamma}^{\alpha} \Xi_{\beta \delta}\left(-p_{i}\right) \Gamma_{(i)}^{\delta}\left(p_{i}\right) .
\end{aligned}
$$

Using (B.8) and (2.12) we can manipulate the last line in (B.13) and express (B.13) as

$$
\begin{aligned}
& \left(p_{i} \cdot k\right)^{-1} \varepsilon_{\mu \nu} p_{i}^{\mu} p_{i}^{\nu} \epsilon_{i, \alpha} \Gamma_{(i)}^{\alpha}\left(p_{i}\right)-\frac{i}{2}\left(p_{i} \cdot k\right)^{-1} \epsilon_{i, \alpha} \varepsilon_{\mu \nu} p_{i}^{\nu} k_{\sigma} \frac{\partial^{2} \mathcal{K}^{\alpha \beta}\left(-p_{i}\right)}{\partial p_{i \sigma} \partial p_{i \mu}} \Xi_{\beta \delta}\left(-p_{i}\right) \Gamma_{(i)}^{\delta}\left(p_{i}\right) \\
& +\frac{i}{4} \epsilon_{i, \alpha} \varepsilon_{b \mu} \frac{\partial^{2} \mathcal{K}^{\alpha \beta}\left(-p_{i}\right)}{\partial p_{i \mu} \partial p_{i b}} \Xi_{\beta \delta}\left(-p_{i}\right) \Gamma_{(i)}^{\delta}\left(p_{i}\right) \\
& -i \epsilon_{i, \alpha}\left(2 p_{i} \cdot k\right)^{-1} \varepsilon_{\mu \nu} p_{i}^{\nu} \frac{\partial \mathcal{K}^{\alpha \beta}\left(-p_{i}\right)}{\partial p_{i \mu}} k_{\rho} \frac{\partial \Xi_{\beta \delta}\left(-p_{i}\right)}{\partial p_{i \rho}} \Gamma_{(i)}^{\delta}\left(p_{i}\right) \\
& +\left(p_{i} \cdot k\right)^{-1} \varepsilon_{\mu \nu} p_{i}^{\mu} p_{i}^{\nu} \epsilon_{i, \alpha} k_{\rho} \frac{\partial}{\partial p_{i \rho}} \Gamma_{(i)}^{\alpha}\left(p_{i}\right) \\
& -\frac{i}{2}\left(p_{i} \cdot k\right)^{-1} k_{a} \varepsilon_{b \mu} \epsilon_{i, \alpha}\left[\left\{p_{i}^{a} \frac{\partial^{2} \mathcal{K}\left(-p_{i}\right)}{\partial p_{i b} \partial p_{i \mu}}-p_{i}^{b} \frac{\partial^{2} \mathcal{K}\left(-p_{i}\right)}{\partial p_{i a} \partial p_{i \mu}}\right\} \Xi\left(-p_{i}\right)\right]_{\gamma}^{\alpha} \Gamma_{(i)}^{\gamma}\left(p_{i}\right) \\
& -\frac{i}{2}\left(p_{i} \cdot k\right)^{-1} k_{a} \varepsilon_{b \mu} \epsilon_{i, \alpha}\left[\frac{\partial \mathcal{K}\left(-p_{i}\right)}{\partial p_{i \mu}}\left\{p_{i}^{a} \frac{\partial \Xi\left(-p_{i}\right)}{\partial p_{i b}}-p_{i}^{b} \frac{\partial \Xi\left(-p_{i}\right)}{\partial p_{i a}}\right\}_{\gamma}^{\alpha} \Gamma_{(i)}^{\gamma}\left(p_{i}\right)\right. \\
& +\left(p_{i} \cdot k\right)^{-1} k_{a} \varepsilon_{b \mu} p_{i}^{\mu} \epsilon_{i, \alpha}\left(J^{a b}\right)_{\gamma}^{\alpha} \Gamma_{(i)}^{\gamma}\left(p_{i}\right) .
\end{aligned}
$$

This can be simplified to

$$
\begin{aligned}
& \left(p_{i} \cdot k\right)^{-1} \varepsilon_{\mu \nu} p_{i}^{\mu} p_{i}^{\nu} \epsilon_{i, \alpha} \Gamma_{(i)}^{\alpha}\left(p_{i}\right)-\frac{i}{4} \epsilon_{i, \alpha} \varepsilon_{b \mu} \frac{\partial^{2} \mathcal{K}^{\alpha \beta}\left(-p_{i}\right)}{\partial p_{i \mu} \partial p_{i b}} \Xi_{\beta \delta}\left(-p_{i}\right) \Gamma_{(i)}^{\delta}\left(p_{i}\right) \\
& +\left(p_{i} \cdot k\right)^{-1} \varepsilon_{\mu \nu} p_{i}^{\mu} p_{i}^{\nu} \epsilon_{i, \alpha} k_{\rho} \frac{\partial}{\partial p_{i \rho}} \Gamma_{(i)}^{\alpha}\left(p_{i}\right)-\frac{i}{2} \varepsilon_{b \mu} \epsilon_{i, \alpha}\left[\frac{\partial \mathcal{K}\left(-p_{i}\right)}{\partial p_{i \mu}} \frac{\partial \Xi\left(-p_{i}\right)}{\partial p_{i b}}\right]_{\gamma}^{\alpha} \Gamma_{(i)}^{\gamma}\left(p_{i}\right) \\
& +\left(p_{i} \cdot k\right)^{-1} k_{a} \varepsilon_{b \mu} p_{i}^{\mu} \epsilon_{i, \alpha}\left(J^{a b}\right)_{\gamma}^{\alpha} \Gamma_{(i)}^{\gamma}\left(p_{i}\right) .
\end{aligned}
$$

The sum of the second and fourth term of (B.15) may be written as

$$
-\frac{i}{4} \epsilon_{i, \alpha} \varepsilon_{b \mu} \frac{\partial^{2}}{\partial p_{i \mu} \partial p_{i b}}\left[\mathcal{K}^{\alpha \beta}\left(-p_{i}\right) \Xi_{\beta \gamma}\left(-p_{i}\right)\right] \Gamma_{(i)}^{\gamma}\left(p_{i}\right)+\frac{i}{4} \epsilon_{i, \alpha} \varepsilon_{b \mu} \mathcal{K}^{\alpha \beta}\left(-p_{i}\right) \frac{\partial^{2} \Xi_{\beta \gamma}\left(-p_{i}\right)}{\partial p_{i \mu} \partial p_{i b}} \Gamma_{(i)}^{\gamma}\left(p_{i}\right) .
$$

Using (B.2) the first term can be shown to be proportional to $\varepsilon_{b \mu} \eta^{b \mu}$ and hence it vanishes due to (2.12). On the other hand the second term vanishes due to (2.17). This allows us 
to express (B.15) as

$$
\begin{aligned}
& \left(p_{i} \cdot k\right)^{-1} \varepsilon_{\mu \nu} p_{i}^{\mu} p_{i}^{\nu} \epsilon_{i, \alpha} \Gamma_{(i)}^{\alpha}\left(p_{i}\right)+\left(p_{i} \cdot k\right)^{-1} \varepsilon_{\mu \nu} p_{i}^{\mu} p_{i}^{\nu} \epsilon_{i, \alpha} k_{\rho} \frac{\partial}{\partial p_{i \rho}} \Gamma_{(i)}^{\alpha}\left(p_{i}\right) \\
& \quad+\left(p_{i} \cdot k\right)^{-1} k_{a} \varepsilon_{b \mu} p_{i}^{\mu} \epsilon_{i, \alpha}\left(J^{a b}\right)_{\gamma}^{\alpha} \Gamma_{(i)}^{\gamma}\left(p_{i}\right) .
\end{aligned}
$$

This proves (2.18).

Open Access. This article is distributed under the terms of the Creative Commons Attribution License (CC-BY 4.0), which permits any use, distribution and reproduction in any medium, provided the original author(s) and source are credited.

\section{References}

[1] S. Weinberg, Photons and gravitons in $S$ matrix theory: derivation of charge conservation and equality of gravitational and inertial mass, Phys. Rev. 135 (1964) B1049 [INSPIRE].

[2] S. Weinberg, Infrared photons and gravitons, Phys. Rev. 140 (1965) B516 [InSPIRE].

[3] D.J. Gross and R. Jackiw, Low-energy theorem for graviton scattering, Phys. Rev. 166 (1968) 1287 [INSPIRE].

[4] R. Jackiw, Low-energy theorems for massless bosons: photons and gravitons, Phys. Rev. 168 (1968) 1623 [INSPIRE].

[5] C.D. White, Factorization properties of soft graviton amplitudes, JHEP 05 (2011) 060 [arXiv:1103.2981] [INSPIRE].

[6] F. Cachazo and A. Strominger, Evidence for a new soft graviton theorem, arXiv:1404.4091 [INSPIRE].

[7] B.U.W. Schwab and A. Volovich, Subleading soft theorem in arbitrary dimensions from scattering equations, Phys. Rev. Lett. 113 (2014) 101601 [arXiv:1404.7749] [INSPIRE].

[8] Z. Bern, S. Davies and J. Nohle, On loop corrections to subleading soft behavior of gluons and gravitons, Phys. Rev. D 90 (2014) 085015 [arXiv: 1405.1015] [INSPIRE].

[9] S. He, Y.-T. Huang and C. Wen, Loop corrections to soft theorems in gauge theories and gravity, JHEP 12 (2014) 115 [arXiv: 1405.1410] [INSPIRE].

[10] A.J. Larkoski, Conformal invariance of the subleading soft theorem in gauge theory, Phys. Rev. D 90 (2014) 087701 [arXiv:1405.2346] [INSPIRE].

[11] F. Cachazo and E.Y. Yuan, Are soft theorems renormalized?, arXiv:1405.3413 [INSPIRE].

[12] N. Afkhami-Jeddi, Soft graviton theorem in arbitrary dimensions, arXiv:1405.3533 [INSPIRE].

[13] J. Broedel, M. de Leeuw, J. Plefka and M. Rosso, Constraining subleading soft gluon and graviton theorems, Phys. Rev. D 90 (2014) 065024 [arXiv:1406.6574] [INSPIRE].

[14] Z. Bern, S. Davies, P. Di Vecchia and J. Nohle, Low-energy behavior of gluons and gravitons from gauge invariance, Phys. Rev. D 90 (2014) 084035 [arXiv:1406.6987] [InSPIRE].

[15] C.D. White, Diagrammatic insights into next-to-soft corrections, Phys. Lett. B 737 (2014) 216 [arXiv:1406.7184] [INSPIRE]. 
[16] M. Zlotnikov, Sub-sub-leading soft-graviton theorem in arbitrary dimension, JHEP 10 (2014) 148 [arXiv: 1407.5936] [INSPIRE].

[17] C. Kalousios and F. Rojas, Next to subleading soft-graviton theorem in arbitrary dimensions, JHEP 01 (2015) 107 [arXiv: 1407.5982] [INSPIRE].

[18] Y.-J. Du, B. Feng, C.-H. Fu and Y. Wang, Note on soft graviton theorem by KLT relation, JHEP 11 (2014) 090 [arXiv:1408.4179] [INSPIRE].

[19] D. Bonocore, E. Laenen, L. Magnea, L. Vernazza and C.D. White, The method of regions and next-to-soft corrections in Drell-Yan production, Phys. Lett. B 742 (2015) 375 [arXiv: 1410.6406] [INSPIRE].

[20] A. Sabio Vera and M.A. Vazquez-Mozo, The double copy structure of soft gravitons, JHEP 03 (2015) 070 [arXiv: 1412.3699] [INSPIRE].

[21] F. Cachazo, S. He and E.Y. Yuan, New double soft emission theorems, Phys. Rev. D 92 (2015) 065030 [arXiv:1503.04816] [INSPIRE].

[22] A.E. Lipstein, Soft theorems from conformal field theory, JHEP 06 (2015) 166 [arXiv: 1504.01364] [INSPIRE].

[23] S.D. Alston, D.C. Dunbar and W.B. Perkins, $n$-point amplitudes with a single negative-helicity graviton, Phys. Rev. D 92 (2015) 065024 [arXiv:1507.08882] [INSPIRE].

[24] Y.-T. Huang and C. Wen, Soft theorems from anomalous symmetries, JHEP 12 (2015) 143 [arXiv: 1509.07840] [INSPIRE].

[25] J. Rao and B. Feng, Note on identities inspired by new soft theorems, JHEP 04 (2016) 173 [arXiv: 1604.00650] [INSPIRE].

[26] F. Cachazo, P. Cha and S. Mizera, Extensions of theories from soft limits, JHEP 06 (2016) 170 [arXiv:1604.03893] [INSPIRE].

[27] A.P. Saha, Double soft theorem for perturbative gravity, JHEP 09 (2016) 165 [arXiv: 1607.02700] [INSPIRE].

[28] A. Luna, S. Melville, S.G. Naculich and C.D. White, Next-to-soft corrections to high energy scattering in QCD and gravity, JHEP 01 (2017) 052 [arXiv:1611.02172] [INSPIRE].

[29] C. Cheung, K. Kampf, J. Novotny, C.-H. Shen and J. Trnka, A periodic table of effective field theories, JHEP 02 (2017) 020 [arXiv: 1611.03137] [INSPIRE].

[30] H. Elvang, C.R.T. Jones and S.G. Naculich, Soft photon and graviton theorems in effective field theory, Phys. Rev. Lett. 118 (2017) 231601 [arXiv:1611.07534] [INSPIRE].

[31] A.P. Saha, Double soft limit of the graviton amplitude from the Cachazo-He-Yuan formalism, Phys. Rev. D 96 (2017) 045002 [arXiv:1702.02350] [INSPIRE].

[32] M. Ademollo et al., Soft dilations and scale renormalization in dual theories, Nucl. Phys. B 94 (1975) 221 [INSPIRE].

[33] J.A. Shapiro, On the renormalization of dual models, Phys. Rev. D 11 (1975) 2937 [INSPIRE].

[34] B.U.W. Schwab, Subleading soft factor for string disk amplitudes, JHEP 08 (2014) 062 [arXiv: 1406.4172] [INSPIRE].

[35] M. Bianchi, S. He, Y.-T. Huang and C. Wen, More on soft theorems: trees, loops and strings, Phys. Rev. D 92 (2015) 065022 [arXiv:1406.5155] [INSPIRE]. 
[36] B.U.W. Schwab, A note on soft factors for closed string scattering, JHEP 03 (2015) 140 [arXiv: 1411.6661] [INSPIRE].

[37] P. Di Vecchia, R. Marotta and M. Mojaza, Soft theorem for the graviton, dilaton and the Kalb-Ramond field in the bosonic string, JHEP 05 (2015) 137 [arXiv:1502.05258] [INSPIRE].

[38] M. Bianchi and A.L. Guerrieri, On the soft limit of open string disk amplitudes with massive states, JHEP 09 (2015) 164 [arXiv:1505.05854] [INSPIRE].

[39] A.L. Guerrieri, Soft behavior of string amplitudes with external massive states, Nuovo Cim. C 39 (2016) 221 [arXiv:1507.08829] [INSPIRE].

[40] P. Di Vecchia, R. Marotta and M. Mojaza, Soft theorems from string theory, Fortsch. Phys. 64 (2016) 389 [arXiv:1511.04921] [InSPIRE].

[41] M. Bianchi and A.L. Guerrieri, On the soft limit of tree-level string amplitudes, arXiv: 1601.03457 [INSPIRE].

[42] P. Di Vecchia, R. Marotta and M. Mojaza, Subsubleading soft theorems of gravitons and dilatons in the bosonic string, JHEP 06 (2016) 054 [arXiv:1604.03355] [INSPIRE].

[43] P. Di Vecchia, R. Marotta and M. Mojaza, Soft behavior of a closed massless state in superstring and universality in the soft behavior of the dilaton, JHEP 12 (2016) 020 [arXiv:1610.03481] [INSPIRE].

[44] A. Strominger, On BMS invariance of gravitational scattering, JHEP 07 (2014) 152 [arXiv: 1312.2229] [INSPIRE].

[45] T. He, V. Lysov, P. Mitra and A. Strominger, BMS supertranslations and Weinberg's soft graviton theorem, JHEP 05 (2015) 151 [arXiv: 1401.7026] [INSPIRE].

[46] A. Strominger and A. Zhiboedov, Gravitational memory, BMS supertranslations and soft theorems, JHEP 01 (2016) 086 [arXiv:1411.5745] [INSPIRE].

[47] S.G. Avery and B.U.W. Schwab, Burg-Metzner-Sachs symmetry, string theory and soft theorems, Phys. Rev. D 93 (2016) 026003 [arXiv: 1506.05789] [InSPIRE].

[48] M. Campiglia and A. Laddha, Asymptotic symmetries of gravity and soft theorems for massive particles, JHEP 12 (2015) 094 [arXiv: 1509. 01406] [INSPIRE].

[49] M. Campiglia and A. Laddha, Sub-subleading soft gravitons: new symmetries of quantum gravity?, Phys. Lett. B 764 (2017) 218 [arXiv:1605.09094] [INSPIRE].

[50] M. Campiglia and A. Laddha, Sub-subleading soft gravitons and large diffeomorphisms, JHEP 01 (2017) 036 [arXiv:1608.00685] [INSPIRE].

[51] E. Conde and P. Mao, BMS supertranslations and not so soft gravitons, JHEP 05 (2017) 060 [arXiv: 1612.08294] [INSPIRE].

[52] T. He, D. Kapec, A.-M. Raclariu and A. Strominger, Loop-corrected Virasoro symmetry of $4 D$ quantum gravity, JHEP 08 (2017) 050 [arXiv:1701.00496] [INSPIRE].

[53] M. Asorey, A.P. Balachandran, F. Lizzi and G. Marmo, Equations of motion as constraints: superselection rules, Ward identities, JHEP 03 (2017) 136 [arXiv: 1612.05886] [INSPIRE].

[54] C. de Lacroix, H. Erbin, S.P. Kashyap, A. Sen and M. Verma, Closed superstring field theory and its applications, Int. J. Mod. Phys. A 32 (2017) 1730021 [arXiv:1703.06410] [InSPIRE]. 
[55] A. Sen, Soft theorems in superstring theory, JHEP 06 (2017) 113 [arXiv:1702.03934] [INSPIRE].

[56] A. Sen and B. Zwiebach, A proof of local background independence of classical closed string field theory, Nucl. Phys. B 414 (1994) 649 [hep-th/9307088] [INSPIRE].

[57] A. Sen and B. Zwiebach, Quantum background independence of closed string field theory, Nucl. Phys. B 423 (1994) 580 [hep-th/9311009] [INSPIRE].

[58] A. Sen, Quantum background independence of closed superstring field theory, work in progress.

[59] G.F. Sterman, An introduction to quantum field theory, Cambridge University Press, Cambridge U.K., (1993) [INSPIRE]. 